\title{
Hepatic Steatosis Index in the Detection of Fatty Liver in Patients with Chronic Hepatitis B Receiving Antiviral Therapy
}

\author{
Jin Won Chang ${ }^{1}$, Hye Won Lee ${ }^{1,2}$, Beom Kyung Kim ${ }^{1,2,3}$, Jun Yong Park ${ }^{1,2,3}$, Do Young Kim ${ }^{1,2,3}$, Sang Hoon Ahn ${ }^{1,2,3}$, \\ Kwang-Hyub Han ${ }^{1,2,3}$, and Seung Up Kim ${ }^{1,2,3}$ \\ ${ }^{1}$ Department of Internal Medicine, Yonsei University College of Medicine, ${ }^{2}$ Yonsei Liver Center, Severance Hospital, and ${ }^{3}$ Institute of \\ Gastroenterology, Yonsei University College of Medicine, Seoul, Korea
}

\section{Article Info}

Received September 7, 2019

Revised December 13, 2019

Accepted December 21, 2019

Published online February 20, 2020

Corresponding Author

Seung Up Kim

ORCID https://orcid.org/0000-0002-9658-8050

E-mail KSUKOREA@yuhs.ac
Background/Aims: The hepatic steatosis index (HSI) is a noninvasive method to assess the severity of hepatic steatosis. Antiviral therapy (AVT) can impact aspartate aminotransferase and alanine aminotransferase levels, which are the main components of the HSI. Thus, we investigated the accuracy of the HSI in detecting hepatic steatosis in patients with chronic hepatitis $B$ $(\mathrm{CHB})$ receiving AVT, compared with those not receiving AVT and in those with nonalcoholic fatty liver disease (NAFLD).

Methods: Patients with CHB or NAFLD who underwent a magnetic resonance imaging proton density fat fraction (MRI-PDFF) evaluation between March 2010 and March 2019 were recruited. Hepatic steatosis was diagnosed when the PDFF exceeded $5 \%$. Area under the receiver operating characteristic curve (AUROC) analysis was used to assess the diagnostic accuracy of the $\mathrm{HSI}$ in the detection of hepatic steatosis.

Results: The mean age of the study population (189 men and 116 women; 244 with CHB [184 with and 60 without AVT] and 61 with NAFLD) was 55.6 years. The AUROC values for detecting hepatic steatosis were similar between patients with $\mathrm{CHB}(0.727 ; p<0.001)$ and those with $\operatorname{NAFLD}(0.739 ; p=0.002)$. However, when patients with $\mathrm{CHB}$ were subdivided into those receiving and not receiving AVT, the AUROC value decreased slightly in patients with $\mathrm{CHB}$ receiving AVT compared to those without not receiving $\operatorname{AVT}(0.707 ; p=0.001$ vs $0.779 ; p=0.001)$.

Conclusions: Despite a slight attenuation, the diagnostic accuracy of the $\mathrm{HSI}$ in patients with $\mathrm{CHB}$ receiving $\mathrm{AVT}$ in detecting hepatic steatosis was still acceptable. Further large-scale studies are required for validation. (Gut Liver 2021;15:117-127)

Key Words: Hepatic steatosis index; Fatty liver; Hepatitis B; Antiviral therapy; Nonalcoholic fatty liver disease

\section{INTRODUCTION}

Nonalcoholic fatty liver disease (NAFLD) is one of the most common causes of chronic liver disease worldwide. ${ }^{1,2}$ With improvements in living standards and lifestyle changes, NAFLD has become increasingly prevalent, occurring in $20 \%$ to $30 \%$ of the general population. ${ }^{3}$ Recent reports indicate that the prevalence of NAFLD in Asian counties is similar to the worldwide occurrence rate, and chronic hepatitis $\mathrm{B}(\mathrm{CHB})$ is a substantial public health concern in these countries. ${ }^{4}$
Accurate assessment of hepatic steatosis is important regardless of the presence or absence of underlying liver disease. The co-existence of hepatic steatosis and CHB may accelerate disease progression, influence the efficacy of antiviral treatment, and increase the risk of developing cirrhosis and hepatocellular carcinoma. ${ }^{5,6}$ Liver biopsy is the gold standard for assessing the severity of hepatic steatosis, but the efficacy and applicability of this procedure is significantly hampered by its invasive nature and the tendency for sampling errors to occur. ${ }^{7,8}$ Although conventional ultrasonography has been widely used as a first- 
line diagnostic tool for hepatic steatosis, there are several factors that diminish the effectiveness of this technique. ${ }^{9-11}$ These include a low sensitivity in detecting hepatic steatosis at an early stage and substantial interoperator discrepancies in the measurements acquired during the imaging.

Recently, controlled attenuation in transient elastography and magnetic resonance imaging-proton density fat fraction (MRI-PDFF) measurements have emerged as accurate, highly reproducible, and noninvasive surrogates in the evaluation of hepatic steatosis in various chronic liver diseases, including NAFLD. ${ }^{12-15}$ However, the high cost of the devices required for these evaluations has limited their widespread clinical use. Accordingly, several noninvasive diagnostic indices based on biochemical laboratory test results or demographic characteristics have been proposed to assess the severity of hepatic steatosis. ${ }^{16}$ Of these indices, the hepatic steatosis index (HSI) is an economical and noninvasive means for assessing the severity of hepatic steatosis with reasonable accuracy. ${ }^{17}$ The constituent variables of the HSI include sex, alanine aminotransferase (ALT), aspartate aminotransferase (AST), body mass index (BMI), and diabetic status. A recent Chinese study reported a greater accuracy of the HSI for detecting biopsy-proven hepatic steatosis when compared to ultrasound (area under the receiver operating characteristic curve [AUROC $]=0.755$ for moderate-to-severe hepatic steatosis and 0.786 for severe hepatic steatosis). ${ }^{18}$ Another study found a significant positive correlation between the HSI and the histopathological grade of hepatic steatosis in patients with chronic hepatitis $\mathrm{C}$ infection. ${ }^{19}$

However, it is not clear whether the acceptable accuracy of the HSI persists in the presence of a therapeutic intervention, which can significantly affect the biochemical markers measured by noninvasive surrogates. Therefore, we investigated the accuracy of the HSI for evaluating hepatic steatosis in patients with $\mathrm{CHB}$ receiving antiviral therapy (AVT) when compared to those not receiving AVT, and to those with NAFLD.

\section{MATERIALS AND METHODS}

\section{Patients}

Between March 2010 and March 2019, patients with CHB or NAFLD who underwent MRI-PDFF evaluations at Severance Hospital, Yonsei University College of Medicine, Seoul, Korea were considered for eligibility in this retrospective, cross-sectional study.

The exclusion criteria were as follows: (1) the presence of other etiologies of chronic liver disease other than $\mathrm{CHB}$ or NAFLD, (2) age $\leq 19$ years, (3) insufficient clinical or laboratory information, (4) insufficient information for calculating HSI, (5) hepatocellular carcinoma or hepatic decompensation at enrollment or a history of either condition, (6) a history of organ transplantation, or (7) significant alcohol consumption (defined as $>21$ drinks per week in men and $>14$ drinks per week in women) ${ }^{20}$

The study protocol was consistent with the ethical guidelines of the Declaration of Helsinki and was approved by the Institutional Review Board of Severance Hospital (IRB number: 4-2019-0639). Informed consent was waived in this study due to its retrospective design.

\section{Estimation of hepatic steatosis severity}

Ultrasonographically diagnosed NAFLD was defined according to ultrasonographic findings such as echogenic or bright liver on imaging consistent with fatty infiltration. ${ }^{21}$ The severity of hepatic steatosis was evaluated using the HSI, which was calculated as follows: $\mathrm{HSI}=8 \times(\mathrm{ALT} /$ AST ratio)+BMI (+2, if female; +2 , if diabetes mellitus). ${ }^{17}$ In addition, hepatic steatosis was also assessed using MRIPDFF and a diagnosis of hepatic steatosis was made when the PDFF exceeded $5 \% .{ }^{22}$

\section{Diagnosis of liver cirrhosis and dyslipidemia}

Liver cirrhosis was diagnosed based on ultrasonographic findings. These included findings such as altered parenchymal echogenicity with coarsened echotexture and surface nodularity, caudate hypertrophy, splenomegaly, and slow portal vein mean flow velocity. ${ }^{23,24} \mathrm{~A}$ diagnosis of dyslipidemia was made when low-density lipoprotein cholesterol was $\geq 160 \mathrm{mg} / \mathrm{dL}$, total cholesterol was $\geq 240 \mathrm{mg} /$ $\mathrm{dL}$, or total triglyceride was $\geq 200 \mathrm{mg} / \mathrm{dL}^{25}$

\section{Statistical analysis}

The patients' demographic and laboratory data were summarized as means \pm standard deviation for continuous variables, and as medians with percentages for categorical variables. Correlation analysis between fat fraction and other variables were conducted using the Pearson method. Mean and frequency data were compared using an independent samples t-test or chi-square test. Logistic binary regression analysis was conducted to identify independent predictors of fatty liver. AUROC analysis was implemented to assess the diagnostic accuracy of the HSI for hepatic steatosis. All statistical analyses were performed using SPSS version 23.0 software (IBM Corp., Armonk, NY, USA). A p-value $<0.05$ was considered statistically significant. 


\section{RESULTS}

\section{Patient characteristics}

Among the 1,437 patients who received MRI-PDFF, 805 patients with other etiologies of chronic liver diseases, other than CHB or NAFLD, were excluded. Further, 327 patients were removed from our study population according to our established exclusion criteria. As a result, a final total of 305 patients were selected for the statistical analysis (Fig. 1).

The baseline characteristics of the study population (189 males and 116 females; 244 with CHB [184 with AVT and 60 without AVT] and 61 NAFLD) are shown in Table 1. The median age, BMI, HSI, and fat fraction as determined by MRI-PDFF of the study participants were 55.6 years, $25.5 \mathrm{~kg} / \mathrm{m}^{2}, 34.7$, and $3.3 \%$, respectively. A total of 83 patients (27.2\%) had fatty liver based on the MRI-PDFF. A total of 36 (14.8\%) patients with CHB had ultrasonographic fatty liver. The respective baseline characteristics of patients with NAFLD and CHB are summarized in Table 1.

\section{Comparison between patients with NAFLD and CHB}

The baseline characteristics of patients with NAFLD and those with $\mathrm{CHB}$ were analyzed and compared (Table 1). Patients with NAFLD were significantly younger (mean: 50.3 years vs 56.9 years) and had a significantly lower proportion of male gender ( $47.5 \%$ vs $65.6 \%)$, a higher BMI (mean: $27.5 \mathrm{~kg} / \mathrm{m}^{2}$ vs $25.0 \mathrm{~kg} / \mathrm{m}^{2}$ ), a higher proportion of diabetes ( $49.2 \%$ vs $23.4 \%$ ), and a lower proportion of liver cirrhosis (52.5\% vs $86.1 \%$ ) than those with CHB (all comparisons $\mathrm{p}<0.05)$. In addition, patients with NAFLD had a lower total bilirubin level (mean: $0.8 \mathrm{mg} / \mathrm{dL}$ vs $1.0 \mathrm{mg} / \mathrm{dL}$ ), a higher AST level (mean: $40.7 \mathrm{U} / \mathrm{L}$ vs $29.1 \mathrm{U} / \mathrm{L}$ ), a higher ALT level (mean: $44.6 \mathrm{U} / \mathrm{L}$ vs $28.0 \mathrm{U} / \mathrm{L}$ ), a higher platelet count (mean: $212 \times 10^{9} / \mathrm{L}$ vs $146 \times 10^{9} / \mathrm{L}$ ), a higher HSI (mean: 38.1 vs 33.8), a higher fat fraction by MRI-PDFF (9.9\% vs $1.6 \%)$, and a higher proportion of fatty liver by MRI-PDFF (62.3\% vs $18.4 \%$ ) than those of patients with CHB (all comparisons $\mathrm{p}<0.05$ ) (Fig. 2).

\section{Comparison between patients with $\mathrm{CHB}$ receiving AVT and those not receiving AVT}

The baseline characteristics of patients with $\mathrm{CHB}$ receiving AVT and those not receiving AVT were compared (Table 1). Patients with CHB receiving AVT were significantly younger (mean: 56.2 years vs 59.2 years) and had a significantly lower BMI (mean: $24.7 \mathrm{~kg} / \mathrm{m}^{2}$ vs $25.9 \mathrm{~kg} / \mathrm{m}^{2}$ ), a higher proportion of liver cirrhosis $(89.7 \%$ vs $75.0 \%)$ and HBeAg positivity (26.4\% vs $5.6 \%$ ), and a lower proportion of fatty liver by MRI-PDFF (14.7\% vs $30.0 \%$ ) than that those not receiving AVT (all comparisons p<0.05) (Fig. 2).

\section{Correlations between fat fraction and other variables}

In the entire study population, the fat fraction as determined by MRI-PDFF showed a significant positive correlation with platelet count, HSI, ALT, BMI, serum albumin, total cholesterol, diabetes, AST, and total protein (all correlations $\mathrm{p}<0.05)$. There was a significant negative correlation between fat fraction as determined by MRI-PDFF and the presence of liver cirrhosis, age, and total bilirubin (all correlations $\mathrm{p}<0.05$ ) (Supplementary Table 1).

In patients with NAFLD, fat fraction as determined by MRI-PDFF showed a significant positive correlation with serum albumin, ALT, total cholesterol, and HSI. In contrast, fat fraction as determined by MRI-PDFF presented with a significant negative correlation with age, hypertension, and presence of liver cirrhosis (all correlations, both positive and negative, $\mathrm{p}<0.05$ ) (Table 2). In patients

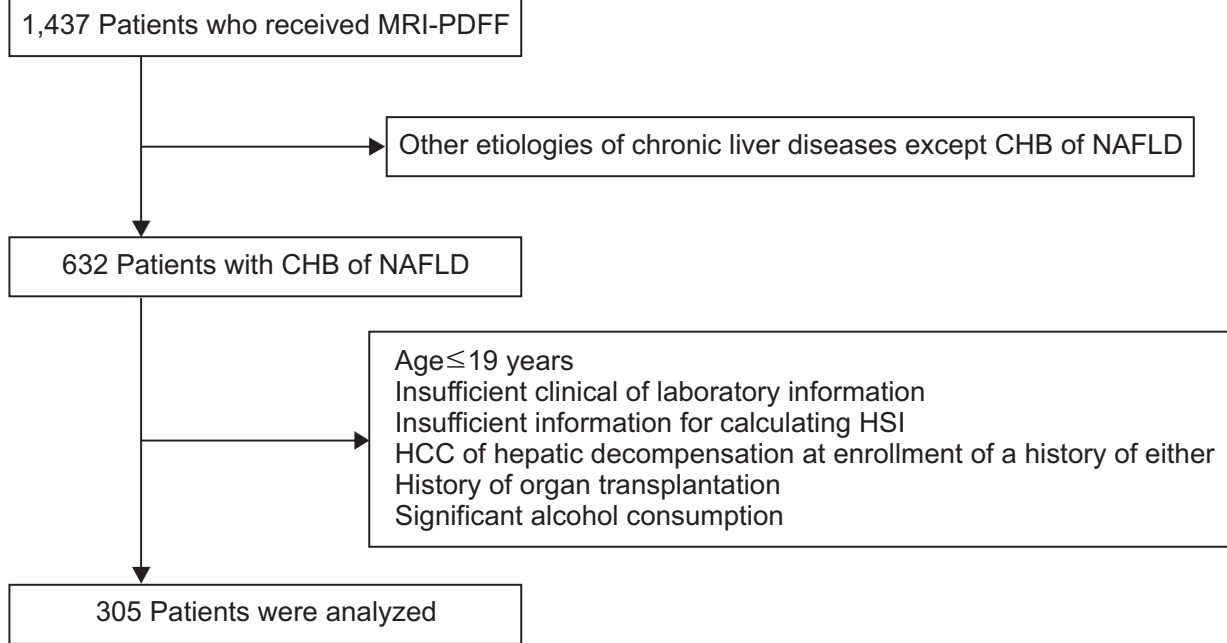

Fig. 1. A graphical representation of the patient selection process. Of 1,437 patients who received MRIPDFF, 305 were selected for the statistical analysis according to our exclusion criteria.

MRI-PDFF, magnetic resonance imaging-proton density fat fraction; CHB, chronic hepatitis B; NAFLD, nonalcoholic fatty liver disease; HSI, hepatic steatosis index; HCC, hepatocellular carcinoma. 


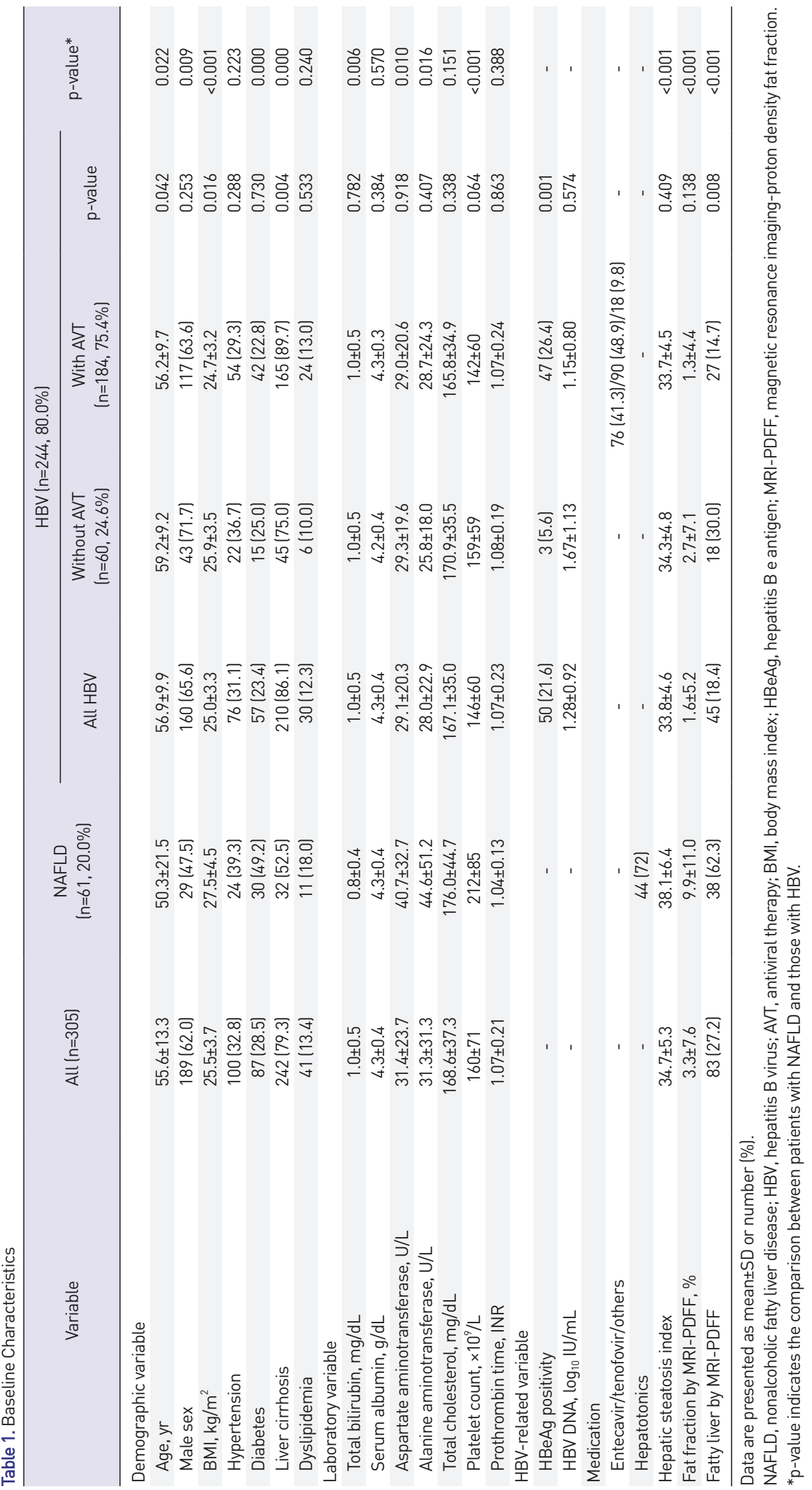




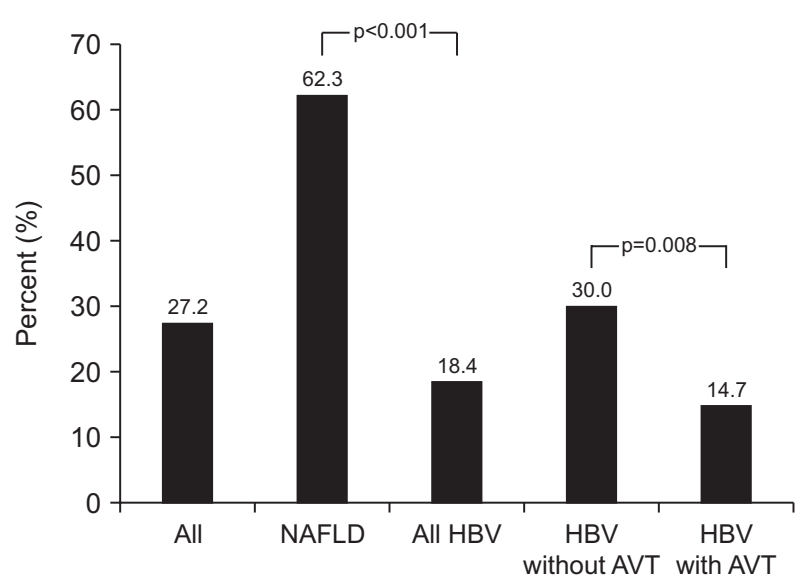

Fig. 2. The prevalence of fatty liver as assessed using MRI-PDFF (magnetic resonance imaging-proton density fat fraction). NAFLD, nonalcoholic fatty liver disease; HBV, hepatitis B virus; AVT, antiviral therapy.

with $\mathrm{CHB}$, fat fraction presented with a significant positive correlation with BMI, presence of dyslipidemia, total cholesterol and HSI, and a significant negative correlation with the presence of liver cirrhosis (all $\mathrm{p}<0.05$ ) (Table 2). The correlation between fat fraction and other variables in patients with CHB receiving AVT and those not receiving AVT are listed in Table 2.

\section{Predictors of the presence of fatty liver}

In entire study population, a higher platelet count (odds ratio $[\mathrm{OR}], 1.012 ; 95 \%$ confidence interval [CI], 1.006 to 1.019 ) and higher HSI (OR, 1.155; 95\% CI, 1.076 to 1.240 ) predicted the presence of fatty liver (all comparisons $\mathrm{p}<0.05$ ) (Supplementary Table 2).

In patients with NAFLD, age, hypertension, and liver cirrhosis were negatively associated with the presence of fatty liver, whereas serum albumin level, platelet count, and HSI were positively associated with the presence of fatty liver in univariate analysis (all $\mathrm{p}<0.05$ ). No predictor of fatty liver was identified in multivariate analysis (Table 3 ).

In all patients with $\mathrm{CHB}$, a higher platelet count (OR, 1.009; 95\% CI, 1.002 to 1.017) and a higher HSI (OR, 1.163; $95 \%$ CI, 1.069 to 1.266$)$ independently predicted the presence of fatty liver $($ all $p<0.05)$ (Table 4$)$. When patients with $\mathrm{CHB}$ were stratified into two groups with and without AVT, a higher HSI was the only predictor of fatty liver in CHB patients without AVT (OR, 1.241; 95\% CI, 1.044 to 1.014; $\mathrm{p}=0.014)$, whereas a higher platelet count (OR, 1.011; 95\% CI, 1.003 to 1.019) and a higher HSI (OR, 1.142; 95\% CI, 1.027 to 1.269 ) independently predicted the presence of fatty liver in $\mathrm{CHB}$ patients receiving AVT (all $\mathrm{p}<0.05$ ) (Table $4)$.

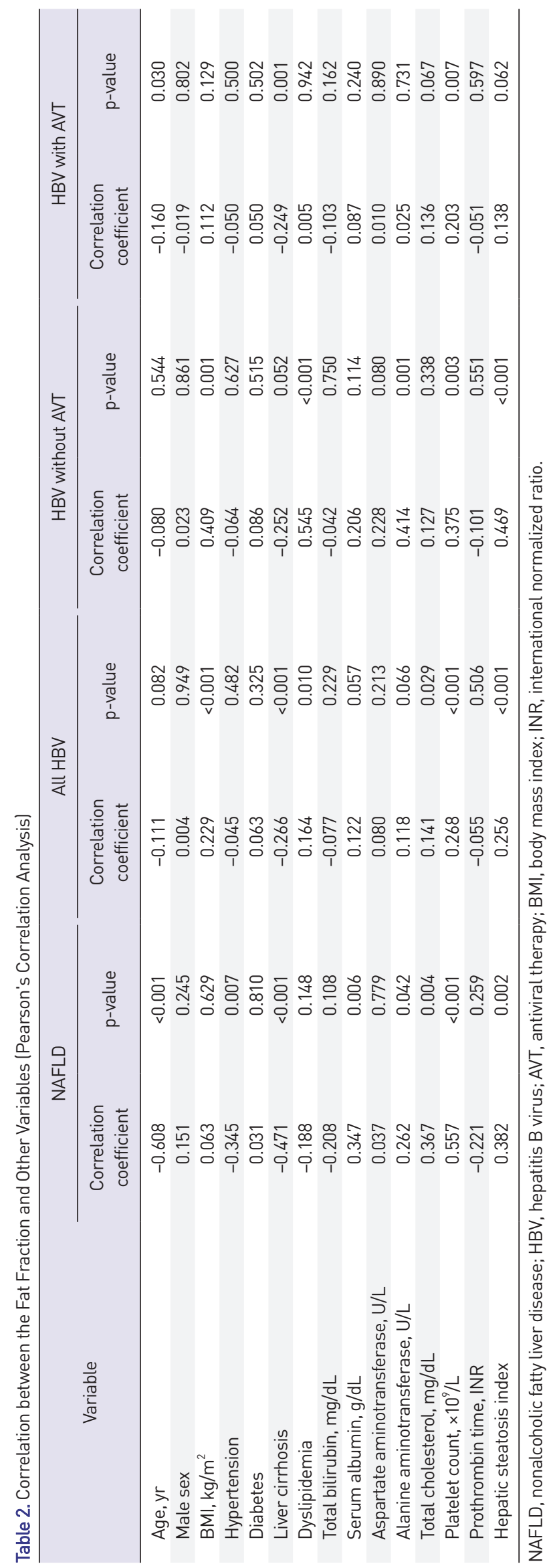

https://doi.org/10.5009/gnl19301 121 
Table 3. Predictors of the Presence of Fatty Liver in Patients with NAFLD

\begin{tabular}{|c|c|c|c|c|}
\hline \multirow{2}{*}{ Variable } & \multicolumn{2}{|c|}{ Univariate } & \multicolumn{2}{|c|}{ Multivariate } \\
\hline & $\mathrm{OR}(95 \% \mathrm{Cl})$ & p-value & $\mathrm{OR}(95 \% \mathrm{Cl})$ & $p$-value \\
\hline Age, yr & $0.920(0.882-0.960)$ & $<0.001$ & $0.972(0.907-1.041)$ & 0.415 \\
\hline Hypertension & $0.230(0.076-0.694)$ & 0.009 & $0.562(0.064-4.943)$ & 0.603 \\
\hline Liver cirrhosis & 0.109 (0.031-0.390) & 0.001 & 0.877 (0.093-8.270) & 0.909 \\
\hline Dyslipidemia & $0.429(0.114-1.612)$ & 0.210 & - & - \\
\hline Total bilirubin, mg/dL & $0.537(0.164-1.753)$ & 0.303 & - & - \\
\hline Serum albumin, g/dL & 33.588 (4.027-280.164) & 0.001 & $9.477(0.293-306.421)$ & 0.205 \\
\hline Platelet count, $\times 10^{9} / \mathrm{L}$ & $1.027(1.013-1.040)$ & $<0.001$ & 1.012 (0.997-1.028) & 0.116 \\
\hline Hepatic steatosis index & $1.211(1.067-1.375)$ & 0.003 & $1.161(0.958-1.407)$ & 0.128 \\
\hline
\end{tabular}

NAFLD, nonalcoholic fatty liver disease; OR, odds ratio; $\mathrm{Cl}$, confidence interval.

\section{Diagnostic accuracy of HSI and other variables in the diagnosis of fatty liver}

The diagnostic accuracy of the HSI was assessed in each subgroup (Table 5, Fig. 3). In NAFLD group, platelet count had the highest AUROC value in the diagnosis of fatty liver (AUROC, 0.886; 95\% CI, 0.793 to 0.978 ), followed by the HSI (AUROC, $0.739 ; 95 \%$ CI, 0.613 to 0.865 ) (both $\mathrm{p}<0.05)$. In CHB group, AUROC value of platelet count was also highest (AUROC, $0.733 ; 95 \% \mathrm{CI}, 0.658$ to 0.808 ), and that of HSI was second (AUROC, 0.727; 95\% CI, 0.649 to 0.805$)$, but the difference of both values was less than that in NAFLD group.

When patients with $\mathrm{CHB}$ were divided into two groups with or without AVT, the AUROC of the HSI in diagnosing fatty liver was increased in patients not receiving AVT (AUROC, 0.779; 95\% CI, 0.651 to 0.907), whereas it was decreased in patients receiving AVT (AUROC, 0.707; 95\% CI, 0.608 to 0.805 ), when compared to the overall AUROC of all patients with $\mathrm{CHB}$ (all comparisons $\mathrm{p}<0.05$ ).

\section{Cutoff value for the HSI in the diagnosis of fatty liver}

The calculated cutoff values of the HSI which maximized the sum of sensitivity and specificity and the proportion of patients with fatty liver are summarized in Table 6. The cutoff values ranged from 33.1 to 33.9 in the subgroups and the HSI accurately predicted fatty liver in $60.3 \%$ to $73.8 \%$ of patients. We then implemented the generally accepted cutoff value of 36 to diagnose fatty liver, ${ }^{17}$ and the changes in diagnostic indices are summarized in Table 6.

\section{DISCUSSION}

Liver biopsy has been regarded as the 'gold standard' for assessing the severity of liver disease. ${ }^{26}$ However, due to its invasive nature and the resultant complications, it has not exhibited a widespread application in clinical practice. ${ }^{27}$ Radiological assessment using ultrasonography, computed tomography, and magnetic resonance imaging have been shown to accurately assess the degree of liver fibrosis or steatosis. ${ }^{28-31}$ However, this noninvasive procedure also has limitations, including the required specialized equipment and high cost of assessment. As such, varying simple and noninvasive tests have been proposed and validated in the identification of patients who are at a high risk of incurring NAFLD. ${ }^{17}$ Of these tests, we focused our investigation on the accuracy of the HSI with simple constituent variables, based on the fat quantity assessed using MRI-PDFF, to diagnose the presence of NAFLD in patients with NAFLD and $\mathrm{CHB}$ with or without $\mathrm{AVT}^{17}$

It is currently accepted that the presence of hepatic steatosis in patients with CHB is significantly associated with a non-physiological response to AVT and accelerated liver fibrosis progression. ${ }^{5,32,33}$ Further, there remains a substantial need for noninvasive predictors of fatty liver in patients with $\mathrm{CHB}$, and recently several noninvasive surrogates, such as the HSI, have been implemented. ${ }^{34}$ However, these surrogate tests rely on biochemical laboratory results, results such as AST or ALT, which are significantly influenced by AVT in patients with $\mathrm{CHB}$. Thus, the diagnostic accuracy of the HSI should be confirmed in patients with CHB undergoing AVT. To this end, the aim of our study was to investigate the diagnostic accuracy of the HSI utilizing NAFLD patients as a control group, and comparing the accuracy of the test between NAFLD patients and those with $\mathrm{CHB}$. In addition, we investigated whether the accuracy of the HSI is altered by AVT in patients with $\mathrm{CHB}$.

In our study, we found that the AUROC values for detecting hepatic steatosis were similar between patients with $\mathrm{CHB}$ and those with NAFLD (0.727 vs 0.739$)$. This indicates that there is good applicability for the HSI in patients with CHB. However, the accuracy of the HSI in NAFLD patients in this study seems relatively low when compared to the original study which proposed the HSI for the diag- 


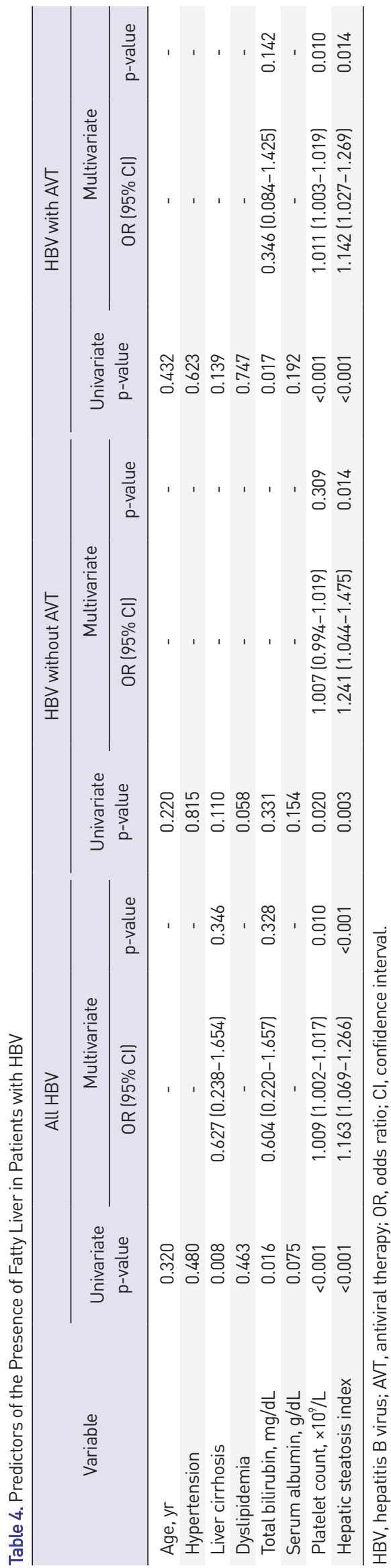

Table 5. Accuracy of the HSI and Other Variables to Diagnose Fatty Liver

\begin{tabular}{lccr}
\hline \multicolumn{1}{c}{ Predictor } & AUROC & $95 \% \mathrm{Cl}$ & p-value \\
\hline NAFLD & & & \\
HSI & 0.739 & $0.613-0.865$ & 0.002 \\
Age, yr & 0.154 & $0.055-0.254$ & $<0.001$ \\
$\quad$ Platelet count, $\times 10^{9} / L$ & 0.886 & $0.793-0.978$ & $<0.001$ \\
All HBV & & & \\
$\quad$ HSI & 0.727 & $0.649-0.805$ & $<0.001$ \\
Age, yr & 0.464 & $0.371-0.557$ & 0.451 \\
Platelet count, $\times 10^{9} / L$ & 0.733 & $0.658-0.808$ & $<0.001$ \\
HBV without AVT & & & \\
HSI & 0.779 & $0.651-0.907$ & 0.001 \\
Age, yr & 0.394 & $0.250-0.537$ & 0.194 \\
Platelet count, $\times 10^{9} / L$ & 0.679 & $0.538-0.821$ & 0.029 \\
HBV with AVT & & & \\
HSI & 0.707 & $0.608-0.805$ & 0.001 \\
Age, yr & 0.465 & $0.341-0.589$ & 0.563 \\
Platelet count, $\times 10^{9} / L$ & 0.744 & $0.652-0.835$ & $<0.001$ \\
\hline
\end{tabular}

$\mathrm{HSI}$, hepatic steatosis index; AUROC, area under the receiver operating characteristic curve; $\mathrm{Cl}$, confidence interval; NAFLD, nonalcoholic fatty liver disease; HBV, hepatitis B virus; AVT, antiviral therapy.

nosis of fatty liver (AUROC, 0.812). ${ }^{17}$ However, when patients with $\mathrm{CHB}$ were subdivided into those with or without AVT, the AUROC value decreased slightly in patients with $\mathrm{CHB}$ receiving AVT compared to those without AVT (0.707 vs 0.779). However, the AUROC values remained within an acceptable range for both groups.

Our study has several clinical implications. First, the prevalence of fatty liver assessed using MRI-PDFF was only $62.3 \%$ in the ultrasonography defined NAFLD group. The accuracy of MRI-PDFF is noted to be highly significant in recent studies, ${ }^{22,35,36}$ and as such this discrepancy in the diagnosis of fatty liver is likely due to false positivity of ultrasonography in cases with increased hepatic parenchymal echogenicity. The coarse parenchymal echogenicity in chronic liver disease is often misdiagnosed as the diffuse increased parenchymal echogenicity in fatty liver. However, as there remains a significant overlap in diagnosing fatty liver between two modalities, no predictor was identified to detect MRI-PDFF-based fatty liver in the ultrasonographically defined NAFLD group.

Second, we found a significant relationship between the presence of hepatic steatosis and platelet count in our study. The platelet count was significantly higher in patients with NAFLD and those with CHB (mean: $212 \times 10^{9}$ / L vs $146 \times 10^{9} / \mathrm{L}$ ). Further, there was a significant correlation between platelet count and fat fraction as determined by MRI-PDFF. After adjustment, the diagnostic value of platelet count remained significant across the entire study population (OR, 1.012) and in those with $\mathrm{CHB}(\mathrm{OR}, 1.009)$. Indeed, platelet count and HSI are the two factors with 

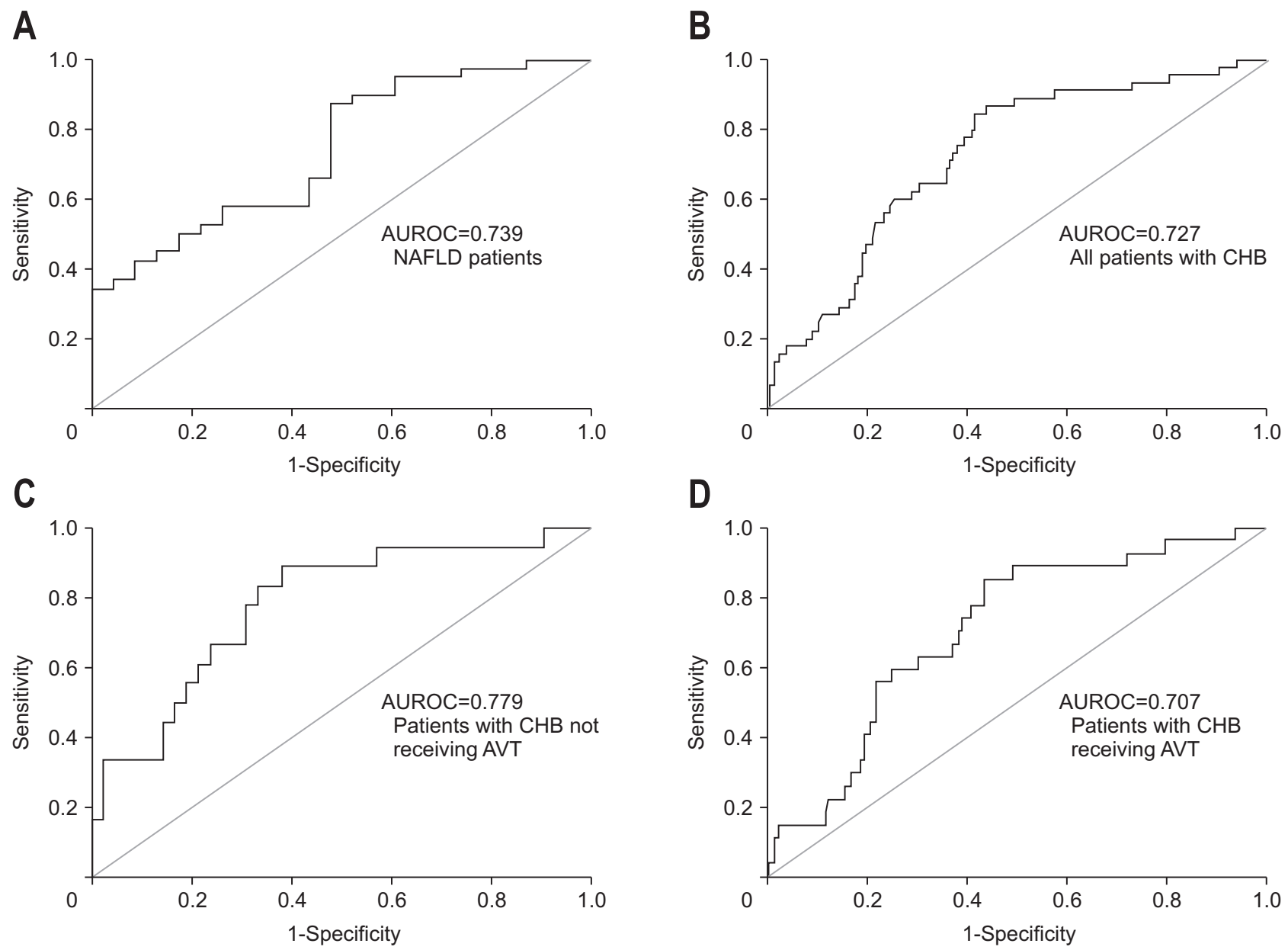

Fig. 3. ROC curves of the HSI for the diagnosis of fatty liver in patients with NAFLD and CHB receiving or not receiving AVT. (A) AUROC=0.739 in NAFLD patients, (B) AUROC $=0.727$ in all patients with $C H B,(C) A U R O C=0.779$ in patients with $C H B$ not receiving $A V T$, and (D) AUROC=0.707 in patients with $\mathrm{CHB}$ receiving AVT.

ROC, receiver operating characteristic; HSI, hepatic steatosis index; NAFLD, nonalcoholic fatty liver disease; CHB, chronic hepatitis B; AVT, antiviral therapy; AUROC, area under the receiver operating characteristic curve.

the highest AUROC values in our study. Our results are supported by findings from previous studies which have demonstrated that platelet count has a positive correlation with hepatic steatosis. ${ }^{37,38}$ In addition, this phenomenon might be associated with the burn-out theory indicating that hepatic steatosis is reduced in advanced fibrosis or cirrhosis stage in the natural history of NAFLD. ${ }^{39}$ Platelets are active participants in the process of liver inflammation, promoting leukocyte recruitment through hepatic sinusoids, and activating effector cells. ${ }^{40}$

Third, we quantified fat content using MRI-PDFF which is the gold standard technique for this measurement. Although other histological features, such as necroinflammation or fibrosis, cannot be detected by MRI-PDFF, MRI-PDFF has been shown to be a precise, accurate, and reproducible noninvasive imaging surrogate for the quantification of liver fat content. ${ }^{41-43}$ Performing a liver biopsy to establish liver fat content is not clinically feasible, and as such our strategy in using the MRI-PDFF for the diagnosis of fatty liver, in the determination of the diagnostic accuracy of the HSI, is particularly relevant.

Finally, in patients with NAFLD, the accuracy of the calculated prediction rate using our own HSI cutoff (33.9) in the diagnosis of fatty liver was higher than that presented previously calculated using a predefined HSI cutoff (36) (73.8\% vs $62.3 \%) .{ }^{17}$ In contrast, in patients with $\mathrm{CHB}$, the correctly predicted rate calculated using our own HSI cutoff (33.4) to diagnose fatty liver was relatively lower than that calculated using the predefined HSI cutoff (36) $(62.7 \%$ vs $71.7 \%$ ). Similar findings were observed in the subgroup analysis of those with CHB undergoing or not undergoing AVT. Although the precise reason for this discrepancy according to the etiology of specific chronic liver disease might be unclear, one of the reasons may be the different proportion of patients with various severity of liver diseases. However, our data suggests that the predefined 
Table 6. Cutoff Value of the HSI Used to Diagnose Fatty Liver

\begin{tabular}{|c|c|c|c|c|}
\hline Diagnostic index & NAFLD & All HBV & $\begin{array}{c}\text { HBV } \\
\text { without AVT }\end{array}$ & $\begin{array}{c}\text { HBV } \\
\text { with AVT }\end{array}$ \\
\hline Patients with fatty liver, No. (\%) & 38 (62.3) & $45(18.4)$ & $18(30.0)$ & $27(14.7)$ \\
\hline Calculated cutoff value & 33.9 & 33.4 & 33.1 & 33.4 \\
\hline Sensitivity, $\%$ & 86.8 & 84.4 & 83.3 & 85.2 \\
\hline Specificity, \% & 52.2 & 57.8 & 61.9 & 56.1 \\
\hline Positive predictive value, $\%$ & 75.0 & 31.2 & 48.4 & 25.0 \\
\hline Negative predictive value, $\%$ & 70.6 & 94.3 & 89.7 & 95.7 \\
\hline Positive likelihood ratio & 1.82 & 2.00 & 2.19 & 1.94 \\
\hline Negative likelihood ratio & 0.25 & 0.27 & 0.27 & 0.26 \\
\hline Correctly predicted, \% & 73.8 & 62.7 & 68.3 & 60.3 \\
\hline \multicolumn{5}{|l|}{ Predefined cutoff value of $\mathrm{HSI}>36$} \\
\hline Patients with HSI >36, No. (\%) & $28(45.9)$ & $78(32.0)$ & $10(16.7)$ & 58 (31.5) \\
\hline Sensitivity, \% & 65.8 & 60.0 & 61.1 & 59.3 \\
\hline Specificity, \% & 54.3 & 74.4 & 78.6 & 73.3 \\
\hline Positive predictive value, $\%$ & 71.4 & 34.6 & 55.0 & 27.6 \\
\hline Negative predictive value, $\%$ & 50.0 & 89.2 & 82.5 & 91.3 \\
\hline Positive likelihood ratio & 1.51 & 2.34 & 2.85 & 2.22 \\
\hline Negative likelihood ratio & 0.61 & 0.54 & 0.49 & 0.56 \\
\hline Correctly predicted, \% & 62.3 & 71.7 & 73.3 & 71.2 \\
\hline
\end{tabular}

HSI, hepatic steatosis index; NAFLD, nonalcoholic fatty liver disease; HBV, hepatitis B virus; AVT, antiviral therapy.

HSI cutoff value of 36 can be used in the diagnosis of fatty liver in patients with $\mathrm{CHB}$, while this value may require some modification to enhance its accuracy in patients with NAFLD. Therefore, we suggest that further validation studies are warranted to establish and confirm the optimal HSI cutoff values for the various etiologies of chronic liver disease.

We are also aware of some limitations of our study which remain unresolved. First, our study has a retrospective design. Thus, the decision to prescribe MRI-PDFF may be different according to the etiology of chronic liver diseases. Indeed, MRI-PDFF could have been performed in patients with NAFLD, whereas it was only done in patients with $\mathrm{CHB}$ as a surveillance imaging technique in the detection of hepatocellular carcinoma. ${ }^{44}$ Accordingly, the overall diagnostic accuracy of HSI in our study might have been significantly influenced due to the high proportion of patients with MRI PDFF-based fatty liver (about $62 \%)$. In addition, the high prevalence of cirrhotic patients might have been caused by selection bias. Indeed, in our study, MRI-PDFF was mostly performed for patients with advanced fibrosis or cirrhosis, as well as those with poor ultrasonographic echo window, or suspicious co-existing hepatic nodules. All of these issues should be resolved in future prospective studies that includes the general population and all patients with full spectrum of the diseases (simple steatosis and NASH in NAFLD patients and inactive and active phases in CHB patients).

Second, our study contains a relatively small sample size. This is particularly true when the study population was divided according to the etiology of chronic liver diseases and AVT status. Large prospective studies will be required to resolve this issue and validate our results.

Third, due to the heterogeneity in the type and duration of hepatotonics, we could not investigate their potential influence on the accuracy of the HSI. In addition, the lack of histological information could be a significant limitation of this study.

In conclusion, the diagnostic accuracy of the HSI in patients with NAFLD was acceptable. Further, despite a slight decrease in accuracy, the diagnostic accuracy of the HSI in patients with CHB receiving AVT was still acceptable in detecting hepatic steatosis. Future larger scale studies will be required for the validation of our results.

\section{CONFLICTS OF INTEREST}

No potential conflict of interest relevant to this article was reported.

\section{ACKNOWLEDGEMENTS}

This study was supported by the Basic Science Research Program through the National Research Foundation of Korea (NRF) funded by the Ministry of Science, ICT \& Future Planning (grant number: 2019R1A2C4070136). 


\section{AUTHOR CONTRIBUTIONS}

Conception: J.W.C., S.U.K. Study design: J.W.C., S.U.K. Participation in patient management and data collection: H.W.L., B.K.K., J.Y.P., D.Y.K., S.H.A., K.H.H., S.U.K. Contributions to the data acquisition, responsible for writing the manuscript, and performing statistical analysis: J.W.C., S.U.K. All authors reviewed the paper and approved the final version. Guarantor: S.U.K.

\section{ORCID}

Jin Won Chang https://orcid.org/0000-0001-9142-7111

Hye Won Lee https://orcid.org/0000-0002-3552-3560

Beom Kyung Kim https://orcid.org/0000-0002-5363-2496

Jun Yong Park https://orcid.org/0000-0001-6324-2224

Do Young Kim https://orcid.org/0000-0002-8327-3439

Sang Hoon Ahn https://orcid.org/0000-0002-3629-4624

Kwang-Hyub Han https://orcid.org/0000-0003-3960-6539

Seung Up Kim https://orcid.org/0000-0002-9658-8050

\section{REFERENCES}

1. Younossi ZM, Koenig AB, Abdelatif D, Fazel Y, Henry L, Wymer M. Global epidemiology of nonalcoholic fatty liver disease: meta-analytic assessment of prevalence, incidence, and outcomes. Hepatology 2016;64:73-84.

2. Loomba R, Sanyal AJ. The global NAFLD epidemic. Nat Rev Gastroenterol Hepatol 2013;10:686-690.

3. Petta S, Muratore C, Craxì A. Non-alcoholic fatty liver disease pathogenesis: the present and the future. Dig Liver Dis 2009;41:615-625.

4. Fan JG, Kim SU, Wong VW. New trends on obesity and NAFLD in Asia. J Hepatol 2017;67:862-873.

5. Ateş F, Yalnız M, Alan S. Impact of liver steatosis on response to pegylated interferon therapy in patients with chronic hepatitis B. World J Gastroenterol 2011;17:4517-4522.

6. Negro F, Clément S. Impact of obesity, steatosis and insulin resistance on progression and response to therapy of hepatitis C. J Viral Hepat 2009;16:681-688.

7. Shackel NA, McCaughan GW. Liver biopsy: is it still relevant? Intern Med J 2006;36:689-691.

8. Emanuele E. Is biopsy always necessary? Toward a clinicolaboratory approach for diagnosing nonalcoholic steatohepatitis in obesity. Hepatology 2008;48:2086-2087.

9. Saadeh S, Younossi ZM, Remer EM, et al. The utility of radiological imaging in nonalcoholic fatty liver disease. Gastroenterology 2002;123:745-750.

10. Hernaez R, Lazo M, Bonekamp S, et al. Diagnostic accuracy and reliability of ultrasonography for the detection of fatty liver: a meta-analysis. Hepatology 2011;54:1082-1090.

11. Schwenzer NF, Springer F, Schraml C, Stefan N, Machann J, Schick F. Non-invasive assessment and quantification of liver steatosis by ultrasound, computed tomography and magnetic resonance. J Hepatol 2009;51:433-445.

12. Reeder SB, Hu HH, Sirlin CB. Proton density fat-fraction: a standardized MR-based biomarker of tissue fat concentration. J Magn Reson Imaging 2012;36:1011-1014.

13. Reeder SB, Robson PM, Yu H, et al. Quantification of hepatic steatosis with MRI: the effects of accurate fat spectral modeling. J Magn Reson Imaging 2009;29:1332-1339.

14. Springer F, Machann J, Claussen CD, Schick F, Schwenzer NF. Liver fat content determined by magnetic resonance imaging and spectroscopy. World J Gastroenterol 2010;16:15601566.

15. Reeder SB, Cruite I, Hamilton G, Sirlin CB. Quantitative assessment of liver fat with magnetic resonance imaging and spectroscopy. J Magn Reson Imaging 2011;34:729-749.

16. Zhang Z, Wang G, Kang K, Wu G, Wang P. Diagnostic accuracy and clinical utility of a new noninvasive index for hepatic steatosis in patients with hepatitis B virus infection. Sci Rep 2016;6:32875.

17. Lee JH, Kim D, Kim HJ, et al. Hepatic steatosis index: a simple screening tool reflecting nonalcoholic fatty liver disease. Dig Liver Dis 2010;42:503-508.

18. Xu L, Lu W, Li P, Shen F, Mi YQ, Fan JG. A comparison of hepatic steatosis index, controlled attenuation parameter and ultrasound as noninvasive diagnostic tools for steatosis in chronic hepatitis B. Dig Liver Dis 2017;49:910-917.

19. Elwan N, Elfert A, Abd-Elsalam S, et al. Study of hepatic steatosis index in patients with chronic HCV infection. Int J Curr Microbiol App Sci 2016;5:266-274.

20. Chalasani N, Younossi Z, Lavine JE, et al. The diagnosis and management of non-alcoholic fatty liver disease: practice Guideline by the American Association for the Study of Liver Diseases, American College of Gastroenterology, and the American Gastroenterological Association. Hepatology 2012;55:2005-2023.

21. Puri P, Sanyal AJ. Nonalcoholic fatty liver disease: definitions, risk factors, and workup. Clin Liver Dis (Hoboken) 2012;1:99-103.

22. Caussy C, Alquiraish MH, Nguyen P, et al. Optimal threshold of controlled attenuation parameter with MRI-PDFF as the gold standard for the detection of hepatic steatosis. Hepatology 2018;67:1348-1359.

23. Gaiani S, Gramantieri L, Venturoli N, et al. What is the criterion for differentiating chronic hepatitis from compensated cirrhosis? A prospective study comparing ultrasonography and percutaneous liver biopsy. J Hepatol 1997;27:979-985.

24. Di Lelio A, Cestari C, Lomazzi A, Beretta L. Cirrhosis: diag- 
nosis with sonographic study of the liver surface. Radiology 1989;172:389-392.

25. Committee for the Korean Guidelines for the Management of Dyslipidemia. 2015 Korean Guidelines for the Management of Dyslipidemia: executive summary (English translation). Korean Circ J 2016;46:275-306.

26. Bravo AA, Sheth SG, Chopra S. Liver biopsy. N Engl J Med 2001;344:495-500.

27. Castéra L, Nègre I, Samii K, Buffet C. Pain experienced during percutaneous liver biopsy. Hepatology 1999;30:15291530.

28. Hamaguchi M, Kojima T, Itoh Y, et al. The severity of ultrasonographic findings in nonalcoholic fatty liver disease reflects the metabolic syndrome and visceral fat accumulation. Am J Gastroenterol 2007;102:2708-2715.

29. Duman DG, Celikel C, Tüney D, Imeryüz N, Avsar E, Tözün N. Computed tomography in nonalcoholic fatty liver disease: a useful tool for hepatosteatosis assessment? Dig Dis Sci 2006;51:346-351.

30. Lee SW, Park SH, Kim KW, et al. Unenhanced CT for assessment of macrovesicular hepatic steatosis in living liver donors: comparison of visual grading with liver attenuation index. Radiology 2007;244:479-485.

31. Fishbein MH, Gardner KG, Potter CJ, Schmalbrock P, Smith MA. Introduction of fast MR imaging in the assessment of hepatic steatosis. Magn Reson Imaging 1997;15:287-293.

32. Jin X, Chen YP, Yang YD, Li YM, Zheng L, Xu CQ. Association between hepatic steatosis and entecavir treatment failure in Chinese patients with chronic hepatitis B. PLoS One 2012;7:e34198.

33. Kim DS, Jeon MY, Lee HW, et al. Influence of hepatic steatosis on the outcomes of patients with chronic hepatitis $\mathrm{B}$ treated with entecavir and tenofovir. Clin Mol Hepatol 2019;25:283-293.

34. Han E, Lee YH, Kim BK, et al. Sarcopenia is associated with the risk of significant liver fibrosis in metabolically unhealthy subjects with chronic hepatitis B. Aliment Pharmacol Ther 2018;48:300-312.
35. Tada T, Kumada T, Toyoda H, et al. Utility of attenuation coefficient measurement using an ultrasound-guided attenuation parameter for evaluation of hepatic steatosis: comparison with MRI-determined proton density fat fraction. AJR Am J Roentgenol 2019;212:332-341.

36. Haufe WM, Wolfson T, Hooker CA, et al. Accuracy of PDFF estimation by magnitude-based and complex-based MRI in children with MR spectroscopy as a reference. J Magn Reson Imaging 2017;46:1641-1647.

37. Fang KC, Cheng YL, Su CW, et al. Higher platelet counts are associated with metabolic syndrome independent of fatty liver diagnosis. J Chin Med Assoc 2017;80:125-132.

38. Cao W, Zhao C, Shen C, Wang Y. Cytokeratin 18, alanine aminotransferase, platelets and triglycerides predict the presence of nonalcoholic steatohepatitis. PLoS One 2013;8:e82092.

39. van der Poorten D, Samer CF, Ramezani-Moghadam M, et al. Hepatic fat loss in advanced nonalcoholic steatohepatitis: are alterations in serum adiponectin the cause? Hepatology 2013;57:2180-2188.

40. Chauhan A, Adams DH, Watson SP, Lalor PF. Platelets: no longer bystanders in liver disease. Hepatology 2016;64:17741784 .

41. Noureddin M, Lam J, Peterson MR, et al. Utility of magnetic resonance imaging versus histology for quantifying changes in liver fat in nonalcoholic fatty liver disease trials. Hepatology 2013;58:1930-1940.

42. Reeder SB. Emerging quantitative magnetic resonance imaging biomarkers of hepatic steatosis. Hepatology 2013;58:1877-1880.

43. Yoo JJ, Kim W, Kim MY, et al. Recent research trends and updates on nonalcoholic fatty liver disease. Clin Mol Hepatol 2019;25:1-11.

44. An C, Kim DY, Choi JY, Han KH, Roh YH, Kim MJ. Noncontrast magnetic resonance imaging versus ultrasonography for hepatocellular carcinoma surveillance (MIRACLEHCC): study protocol for a prospective randomized trial. BMC Cancer 2018;18:915. 